\title{
Dose-dependent Misrejoining of Radiation-Induced DNA Double-Strand Breaks in Human Fibroblasts: Experimental and Theoretical Study for high and low LET Radiation.
}

Bjorn Rydberg, Brian Cooper, Priscilla K. Cooper, William Holley and Aloke Chatterjee.

Lawrence Berkeley National Laboratory, Life Sciences Division, One Cyclotron Road, Berkeley, California 94720.

Number of Figures: 6

Number of Tables: 0

Running Title: DNA Double-strand Break Misrejoining

Corresponding author:

Björn Rydberg

Lawrence Berkeley National Laboratory

Building 74 - 157

One Cyclotron Road

Berkeley, CA 94720

Telephone: (510) 486-7045

Fax: (510) 486-6816

E-mail: berydberg@lbl.gov 


\section{ABSTRACT}

Rydberg, B., Cooper, B., Cooper, P. K., Holley, W. and Chatterjee, A. Dosedependent Misrejoining of Radiation-Induced DNA Double-Strand Breaks in Human Fibroblasts: Experimental and Theoretical Study for high and low LET Radiation. Radiat. Res.

Misrejoining of DNA double-strand breaks (DSBs) was measured in human primary fibroblasts after exposure to X-rays and high LET particles ( $\mathrm{He}, \mathrm{N}$ and $\mathrm{Fe}$ ) in the dose range $10-80 \mathrm{~Gy}$. To measure joining of wrong DNA ends, the integrity of a $3.2 \mathrm{Mbp}$ restriction fragment was analyzed directly after exposure and after $16 \mathrm{hr}$ of repair incubation. It was found that the misrejoining frequency for X-rays was non-linearly related to dose, with less probability of misrejoining at low doses than at high doses. The dose dependence for the high LET particles, on the other hand, was closer to being linear, with misrejoining frequencies higher than for X-rays particularly at the lower doses. These experimental results were simulated with a Monte-Carlo approach that includes a cell nucleus model with all 46 chromosomes present, combined with realistic track structure simulations to calculate the geometrical positions of all DSBs induced for each dose. The model assumes that the main determinant for misrejoining probability is the distance between two simultaneously present DSBs. With a Gaussian interaction probability function with distance, it was found that both the low and high LET data could be fitted with an interaction distance (sigma of the Gaussian curve) of $0.25 \mu \mathrm{m}$. This is half the distance previously found to best fit chromosomal aberration data in human lymphocytes using the same methods (Holley et al. Radiat. Res . 158, 568-580 (2002)). The discrepancy may indicate inadequacies in the chromosome model, for example insufficient chromosomal overlap, but may also partly be due to differences between fibroblasts and lymphocytes. Although the experimental data was obtained at high doses, the Monte Carlo calculations could be extended to lower doses. It was found that a linear component of misrejoining versus dose dominated for doses below 1 Gy for all radiations, including X-rays. The calculated relative biological efficiency (RBE) for misrejoining at this low dose region was 31 for the He-ions, 28 for the $\mathrm{N}$-ions and 19 for Fe-ions. 


\section{INTRODUCTION}

A major class of deleterious effects induced by ionizing radiation is believed to be a consequence of DNA double-strand breaks (DSBs). In particular when such breaks are joined with wrong DNA ends during a subsequent nonhomologous endjoining event, the results may be deletions, chromosome aberrations and reproductive cell death. Misrejoining (here defined as joining of wrong DNA ends) can be studied with a technique that measures the integrity of a defined large restriction fragment $(1,2)$. When such measurements are carried out with X-rays with doses of 80 Gy and above, a surprisingly large fraction of around $35 \%$ of DSBs is found to misrejoin. This contrasts with the frequency of chromosome aberrations, which at smaller doses of 10 Gy or below corresponds to about $1-2 \%$ of the frequency of DSBs (discussed in (3)). A high proportion of misrejoining is also found when the technique of radiation hybrid (RH) mapping is carried out for the purpose of genomic mapping (4). The number of random DNA fragment insertions achieved with this technique is fully compatible with a $35 \%$ misrejoining frequency at the very high doses used (often above $200 \mathrm{~Gy}$ ).

To reconcile the frequency of misrejoining at high doses with chromosome aberration data at lower doses, Radivoyevitch et al. (3) proposed that misrejoining is dependent on the distance between initially induced DSBs, and that the $35 \%$ value seen at $80 \mathrm{~Gy}$ and above is a saturation value. The mathematical modeling consisted in dividing up the cell nucleus into small compartments and postulating, as an approximation, that DSB ends generated within the same compartment could join with any other DNA end within that compartment with the same probability (including the correct end), while joining of ends between compartments was excluded. To fit the existing data at high doses, it was also needed to assume that only $35 \%$ of the DSBs were available for misrejoining and the remaining $65 \%$ of the breaks were always correctly joined, thus generating a maximum saturation value of $35 \%$ at high doses. With this model it was

necessary to divide the cell nucleus into approximately $10^{3}$ compartments in order to obtain a reasonable misrejoining frequency at lower doses, which, on a linear scale corresponds to $1 / 10$ of the size of the nucleus. Thus, for a nucleus of $5 \mu \mathrm{m}$ radius, breaks 
would need to be within about $0.5 \mu \mathrm{m}$ in order to have a possibility to misrejoin. This happens frequently at high doses, but is infrequent at lower doses with a Poisson statistical distribution. A value of $0.5 \mu \mathrm{m}$ compares favorably with the generalized model of dual radiation action (5), where a pair of lesions within similar distances is assumed to cause biological effects.

In subsequent work it was found that the saturation value for misrejoining apparently is similar for high LET radiation as for low LET radiation (6) and that it is also similar in different parts of the genome and in different cell types (2). With an improved analysis of the gel profiles (2), the percentage of correctly joined breaks at saturation was found to be $55 \%$, somewhat lower than found previously. This value is used in the present paper. It is presently not known why $55 \%$ of the DSBs always join correctly, but it has recently been shown that about 30\% of DSBs as detected by present techniques are generated during the lysis procedure and are not actually present in cells as DSBs but rather as heat-labile sites $(7,8)$. It is also possible that the remainder of DSBs that are not available for misrejoining are somehow hidden without causing chromatin breakage.

Measurement of misrejoining at low doses is a challenge because of the limited sensitivity of the assay. However, Löbrich et al. (9) used fractionated small doses with 24 hr intervals to simulate the low dose situation. Using human fibroblasts that stay metabolically normal in culture without undergoing apoptosis long times after irradiation, they could demonstrate a dose dependence of misrejoining frequency for X-rays that qualitatively agrees with the values expected from modeling. Furthermore, it was found that the situation is different for high LET radiation (alpha particles) (10), in which case no dose dependence could be demonstrated down to 10 Gy of fractionated doses (total 80 Gy). This could be understood by the assumption that intra-track DSBs were mainly responsible for misrejoining in this case, since the distances between intra-track DSBs do not change with dose.

In the present paper we report measurements of misrejoining using single doses in the dose range of 10-80 Gy for X-rays and three different high LET particle radiation. We find a prominent dose dependence for X-rays, and only a slight dose dependence for the high LET particles, in agreement with the previous study. We also use a realistic 
computational procedure that uses Monte Carlo calculations of particle tracks in a computer model that incorporates all 46 chromosomes in a nucleus in the form of random coils with volume constraints (11). The model assumes that the misrejoining probability is a Gaussian function with distance between breaks. The best fit to the present data for human fibroblasts is obtained with a misrejoining interacting distance of $0.25 \mu \mathrm{m}$ (standard deviation of the Gaussian function), which is somewhat smaller than a previous estimate of the misrejoining interaction distance used for estimation of chromosomal aberrations in human lymphocytes (11).

\section{MATERIALS AND METHODS}

\section{Cell Culture}

Primary normal human dermal fibroblasts, GM38, were obtained from the Human Genetic Mutant Cell Repository, Coriell Institute for Medical Research. The cells were grown in humidified $5 \% \mathrm{CO}_{2}$ at $37^{\circ} \mathrm{C}$ in McCoy's $5 \mathrm{~A}$ medium supplemented with $10 \%$

fetal bovine serum (GIBCO), 2mM glutamine, $10 \mathrm{mM}$ Hepes, and antibiotic/antimycotic solution (penicillin at 100 units $/ \mathrm{ml}$, streptomycin sulfate at $100 \mu \mathrm{g} / \mathrm{ml}$, amphotericin B at $0.25 \mu \mathrm{g} / \mathrm{ml}$ ) (GIBCO). Early passage GM38 cells were grown to confluence prior to all experiments, at which time they were nearly exclusively in the G0 or G1 stages of the cell cycle.

\section{Irradiation and Dosimetry}

Cell monolayers were washed in PBS and irradiated in PBS at $0^{\circ} \mathrm{C}$. X-ray exposures were performed with a Pantax $320 \mathrm{kVp}$ X-ray unit fitted within a lead cabinet (Willick Engineering). The X-rays were filtered through $0.5 \mathrm{~mm} \mathrm{Cu}$ at a dose rate of 2-3

Gy/min. X-ray dosimetry was carried out with a $0.18 \mathrm{~cm}^{3}$ calibrated ion chamber with temperature and pressure correction (Radcal Corporation). $29 \mathrm{MeV} / \mathrm{u} \mathrm{N}$ ions with LET 97 $\mathrm{keV} / \mu \mathrm{m}$ and $7 \mathrm{MeV}$ He ions with LET $74 \mathrm{keV} / \mu \mathrm{m}$ were obtained at dose rates of 50-100 Gy/min from the 88-Inch Cyclotron at the Lawrence Berkeley National Laboratory. Dosimetry was performed with ion chambers as described previously (12). The energy calibration and special precautions used for the $7 \mathrm{MeV}$ He ions are described in detail in 
(13). Exposures to $1 \mathrm{GeV} / \mathrm{u} \mathrm{Fe}$ ions were carried out at Brookhaven National Laboratory using the Alternating Gradient Synchrotron with dosimetry as described by Zeitlin et al. (12). After irradiation with the various sources, the cell cultures were either harvested directly or incubated at $37^{\circ} \mathrm{C}$ for $16 \mathrm{hrs}$ to allow for repair.

\section{Standard FAR assay}

To measure total breaks remaining unrejoined after repair, pulsed-field gel electrophoresis was carried out essentially as described previously (7). Briefly, ${ }^{14} \mathrm{C}-\mathrm{TdR}$ labeled cells were embedded in agarose plugs and lysed in $0.4 \mathrm{M} \mathrm{Na}_{2}$ EDTA, $1 \%$ sarkosyl, $1 \mathrm{mg} / \mathrm{ml}$ Proteinase $\mathrm{K}, \mathrm{pH} 8.0$ at $50{ }^{\circ} \mathrm{C}$ for $24 \mathrm{hrs}$. Pulsed-field gel electrophoresis was performed using a CHEF-DR II apparatus (Bio-Rad) with a switch time of $1 \mathrm{hr}$ for a total run time of $20 \mathrm{hrs}$ at $1.5 \mathrm{~V} / \mathrm{cm}$ at $14{ }^{\circ} \mathrm{C}$. Under these conditions, high molecular weight DNA up to a size of approximately $7 \mathrm{Mbp}$ is eluted from the plug in the form of a compression band $(14,15)$. After electrophoresis, the gels were cut into slices comprising the plug and the compression band and the fraction of total radioactivity present in the compression band in each lane (FAR) was measured by liquid scintillation. To calculate percent breaks remaining after repair, the induction curve for FAR versus dose was measured and used as calibration.

Preparation of agarose plugs containing large DNA molecules for the hybridization assay.

The cells were trypsinized and resuspended in PBS at a concentration of approximately $1.5 \times 10^{7}$ cells $/ \mathrm{ml}$. Equal volumes of $1.5 \%$ low gelling temperature agarose (Type VII, Sigma) in PBS were added to the cell suspensions and the mixtures were poured into agarose plugs with dimensions of $20 \times 9 \times 1.2 \mathrm{~mm}$. The plugs were allowed to gel by cooling on ice for ten min and then immersed into a lysis solution $(0.4 \mathrm{M}$ $\mathrm{Na}_{2}$ EDTA, $1 \%$ sarkosyl, $1 \mathrm{mg} / \mathrm{ml}$ Proteinase $\left.\mathrm{K}, \mathrm{pH} 8.0\right)$ for $1 \mathrm{hr}$ at $0^{\circ} \mathrm{C}$ followed by at least 48 hours at $50^{\circ} \mathrm{C}$ with a change to fresh lysis buffer after 24 hours. The plugs were then stored in TE (10 mM Tris, $1 \mathrm{mM} \mathrm{EDTA})$ at $4^{\circ} \mathrm{C}$ until treatment with restriction enzymes. 
Restriction digest and pulsed-field gel electrophoresis.

Plugs with DNA were additionally washed twice in 100x volume TE for a total of at least two hours and one time for at least one hour in NotI restriction enzyme buffer (Promega) directly prior to restriction digest. Pieces corresponding to $1 / 3$ of the original plugs were then transferred to $1.5 \mathrm{ml}$ Eppendorf tubes containing $0.25 \mathrm{ml}$ of the restriction buffer and 17 units of restriction enzyme (NotI, Promega). The plugs were incubated overnight at $37^{\circ} \mathrm{C}$, washed for one hour in TE, and loaded into a pulsed-field gel. Pulsed-field gel electrophoresis was carried out using a CHEF-DR II apparatus (BioRad). Gels were made from $0.8 \%$ UltraPure agarose (BioRad) in $0.5 \mathrm{X}$ TBE ( $1 \mathrm{X}$ TBE is $89 \mathrm{mM}$ Tris, $89 \mathrm{mM}$ boric acid, $2 \mathrm{mM} \mathrm{Na} 2 \mathrm{EDTA}, \mathrm{pH} \mathrm{8.0)}$. The gels were run at $14^{\circ} \mathrm{C}$ in $0.5 \mathrm{X}$ TBE for 140 hours at $50 \mathrm{~V}$ with a switch time changing linearly from $50 \mathrm{~s}-5000 \mathrm{~s}$ (16). After electrophoresis, gels were stained in $0.5 \mu \mathrm{g} / \mathrm{ml}$ ethidium bromide and photographed with a CDC camera on a UV trans-illuminator.

\section{Southern Blot and Hybridization Protocol}

The procedure was essentially as described by Lobrich et al. (1) and later modified by Fouladi et al. (2). After electrophoresis, the DNA in the gel was depurinated in $0.25 \mathrm{M} \mathrm{HCl}$ for 15 minutes, and then vacuum-blotted for 1.5 hours in an alkaline solution $(0.5 \mathrm{M} \mathrm{NaOH})$ onto Amersham's Hybond-N+ charged nylon membrane. Vacuum blotting was done with an LKB VacuGene unit (Pharmacia LKB Biotechnology) according to the manufacturer's instructions. After the transfer, the membrane was washed in $2 \mathrm{X} \mathrm{SSC}$ ( $20 \mathrm{X} \mathrm{SSC}$ is $3 \mathrm{M} \mathrm{NaCl}, 0.3 \mathrm{M} \mathrm{Na}_{3}$ citrate) for $15 \mathrm{~min}$, wrapped in plastic wrap and stored at $4^{\circ} \mathrm{C}$ for subsequent analysis.

For hybridization, moist nylon membranes were placed, sample side inward, into a Hoefer hybridization tube containing $20 \mathrm{ml}$ of hybridization buffer; 5X SSPE (20 X SSPE is $3.6 \mathrm{M} \mathrm{NaCl}, 0.2 \mathrm{M}$ Sodium Phosphate, $0.02 \mathrm{M}$ EDTA pH 7.7), 5X Denhardts (50 X Denhardts is $5 \mathrm{~g}$ Ficoll, $5 \mathrm{~g}$ of polyvinylpyrrolidine, $5 \mathrm{~g}$ of bovine serum albumin in $500 \mathrm{mls} \mathrm{H}_{2} \mathrm{O}$ ), $0.5 \%$ sodium dodecyl sulfate (SDS), and $0.25 \mathrm{mg} / \mathrm{ml}$ heat denatured salmon sperm DNA. The membranes were prehybridized for at least 3 hours in a Hoefer HB 1100D Red Roller II hybridization oven at $65^{\circ} \mathrm{C}$. Following prehybridization, the hybridization buffer was drained from the tube and replaced with $20 \mathrm{ml}$ fresh 
hybridization buffer containing $25 \mathrm{ng}$ of heat denatured, ${ }^{32} \mathrm{P}$-labeled probe specific for a 3.2 Mbp NotI fragment on chromosome 21 (16). The probe was labeled by randompriming using an Amersham MegaPrime DNA labeling kit according to the manufacturer's instructions with $6000 \mathrm{Ci} / \mathrm{mmol} \quad \alpha-{ }^{32} \mathrm{P} \quad \mathrm{dCTP}$ (Amersham). Unincorporated nucleotides were removed from the labeled DNA solution with a G-50 Quick Spin column (Boehringer Mannheim). At least $10^{8}$ total counts of activity were added to the hybridization buffer. The membrane was hybridized overnight at $65^{\circ} \mathrm{C}$, followed by 3 washes at $65^{\circ} \mathrm{C}$ for $45 \mathrm{~min}$ each in $100 \mathrm{ml}$ of $2 x \mathrm{SSPE}, 0.1 \% \mathrm{SDS}$, one wash for $30 \mathrm{~min}$ at $65^{\circ} \mathrm{C}$ in $1 \mathrm{xSSPE}, 0.1 \% \mathrm{SDS}$, followed by two washes for $30 \mathrm{~min}$ at $65^{\circ} \mathrm{C}$ in $1 \%$ SDS, $1 \mathrm{mM}$ EDTA, $40 \mathrm{mM}$ sodium phosphate $\mathrm{pH}$ 7.2. The membrane was wrapped in plastic wrap and exposed to a Molecular Dynamics phosphor screen for at least 16 hours. The screen was scanned using the PhosphorImager SI scanner from Molecular Dynamics to produce a digital image of the radioactivity on the membrane.

\section{Data evaluation.}

The PhosphorImager pictures were analyzed as described in detail in (2) with the use of software provided by the manufacturer. Briefly, each lane was individually scanned to give the radioactivity signal as a function of electrophoresis distance. The profiles had a peak at the location of the intact 3.2 Mbp NotI fragment, and a smear below the peak corresponding to the sum of broken fragments and misrejoined fragments. Since the average NotI restriction fragment is much smaller than $3.2 \mathrm{Mbp}$, misrejoined NotI fragments above 3.2 Mbp were rarely seen and were not included in the evaluation. A background signal was determined at long electrophoresis distances and assumed to be constant in the whole lane. After subtraction of the constant background, the total area in the lane including the $3.2 \mathrm{Mbp}$ peak and the smear was determined $\left(\mathrm{A}_{\text {tot }}\right)$, excluding any signal above the peak (which was variable between experiments). A straight line was then drawn by hand to divide the peak from the underlying smear (as shown in Figure 2B). The rational for this method is illustrated in Figure $2 \mathrm{C}$ and 2D, which show how a theoretical distribution is modified by the limited resolution of the gel. In this situation a straight line, as shown, is an excellent approximation in the division of the areas in peak and smear. However, the step function in Figure $2 \mathrm{C}$ is only a rough approximation of the 
smear distribution, which is complex and not known for high LET radiation. The MonteCarlo calculations (see below) therefore include a correction by comparing the calculated smear distributions with the simplified assumptions made in the analysis of the experimental data. The area of the peak above he straight line corresponding to the intact 3.2 Mbp fragments was then calculated $\left(\mathrm{A}_{\text {peak }}\right)$. By assuming random induction of DSBs and Poisson statistics, the sum of broken and misrejoined DSBs per 3.2 Mbp was then calculated as $-\ln \left(\mathrm{A}_{\text {peak }} / \mathrm{A}_{\text {tot }}\right)$. The average value for non-irradiated controls in each experimental series was then subtracted from the values of irradiated samples to give the final value due to irradiation.

\section{Monte-Carlo calculations}

These calculations are based on a model which explicitly takes into account interphase $\left(\mathrm{G}_{0} / \mathrm{G}_{1}\right)$ chromosome structure, nuclear organization of chromosomes, the production of double strand breaks, and the subsequent rejoining events in a faithful or unfaithful manner (11). Each of the 46 chromosomes for a human cell is modeled as a random polymer inside a spherical volume. The chromosome spheres are packed randomly inside a spherical nucleus with an allowed overlap controlled by a parameter $\Omega$. Random ionizing particle trajectories are simulated with Monte-Carlo approach until the desired dose has been reached. Many such simulations are combined to give the desired statistical accuracy.

In order to calculate the yield and location of double strand breaks we use our existing track structure code that is based on the microscopic distribution of energy depositions around the trajectory of a charged-particle track and includes the ensuing radiation chemistry that evolves due to the radiolysis of water contained in a cellular environment. The details of the calculations for estimating double strand break yields including their respective locations in the genome have been described in an earlier publication (11). Both the radical mechanism involving water radicals and direct deposition of energy on the various DNA sites are included to calculate the sugar and base damages. A double-strand break (DSB) is defined as single-strand breaks on opposite strands with maximum separation of $10 \mathrm{bp}$. Nearly $90 \%$ of all correlated singlestrand breaks are formed within $10 \mathrm{bp}$ with an additional 5\% in the interval 10-20 base- 
pairs. The choice of separation distance is therefore not crucial. Their exact locations in the chromatin fiber are recorded which then allow us to determine the positions within the cell of single strand breaks with associated base damages or double strand breaks with associated base damages and sugar damages. The damage clusters at double-strand breaks (which we treat as single entities for rejoining purposes) are defined as a region of DNA containing one or more DSBs with no internal region of $20 \mathrm{bp}$ or more of contiguous undamaged base pairs. Once the locations of the double strand breaks are determined their rejoining is simulated by a Monte Carlo procedure which uses a Gaussian proximity function given by

$$
\text { Probability } \approx \exp \left(-\mathrm{d}^{2} / 2 \sigma^{2}\right)
$$

Where $d$ is the initial distance between any two DNA ends and the range parameter $\sigma$ is the standard deviation of the range of interaction of two ends. In our model, we have two parameters, $\sigma$ and $\Omega$, which are adjustable. Instead of varying both the parameters we have used the overlap parameter, $\Omega$, as constant and assumed to be $7 \%$ on an average irrespective of the cell line. We have fitted the data by varying $\sigma$ and it is variable for different cell lines. For the primary human fibroblasts used in this work, the value 0.25 $\mu \mathrm{m}$ seem to fit the data quite well within the error bars. In our calculations we use the experimental observations (2) that at high doses, the faithful rejoining fraction reaches a plateau value of about $55 \%$ of the initial number of DSBs. Thus we choose $55 \%$ of the breaks at random and assign them to be joined faithfully. These breaks include the heatlabile sites that were not real breaks in the cell but which became breaks during the $50^{\circ} \mathrm{C}$ lysis step (7). In our Monte-Carlo approach we assume that all sugar damage results in breaks, thus including the heat-labile sites that resulted in breaks during lysis (11). The rejoining fidelity of the remaining $45 \%$ is decided based on proximity function. The rejoining algorithm proceeds as follows: (1) Relative probabilities of rejoining between all unrejoined ends are calculated using Gaussian expression above (2). The probabilities are normalized to unity by dividing by the sum of all probabilities. (3) A rejoining interaction between two ends is selected by means of a random number distributed uniformly between 0 to 1.0 (4). The two joined ends are removed from the set of unjoined 
ends and the procedure is repeated until all ends with non-zero rejoining probabilities have joined. Using this procedure, occasionally, isolated or "orphan" ends will remain unjoined if a limiting interaction range has been introduced into the definition of proximity function. This fraction is generally less than a few percent. Calculations were performed for $1 \mathrm{GeV} / \mathrm{n}$ Fe ions, $30 \mathrm{MeV} / \mathrm{n} \mathrm{N}$ ions, 1 and $2 \mathrm{MeV} / \mathrm{n} \mathrm{He}$ ions and $10 \mathrm{keV}$ electrons to simulate $\mathrm{X}$-rays.

After completion of the calculations described above for a range of doses and parameter $\sigma$, random 3.2 Mbp segments were introduced in the genome to simulate the 3.2 Mbp restriction fragment studied experimentally. Misrejoining events within such segments were recorded, to give the total frequency of misrejoining. However, not all events are detected experimentally with the data evaluation method used for the gel profiles, for example inversions and small deletions within the $3.2 \mathrm{Mbp}$ fragment will not change the size sufficiently to be detected. Also, the experimental data were evaluated using the Poisson approximation, which is correct only for randomly induced breaks. To simulate the experimental values, two corrections were performed to the calculated values. First, events that produced fragments within the range of 3.0 and 3.4 Mbp (in excess of those produced from a random distribution) were subtracted. This correction compensates for the way the experimental gel profiles were divided into peak and smear area with a straight line (Figure 2B), which is a good approximation for randomly induced breaks but not for excess events that produce very small changes in size. Secondly, a Poisson assumption was applied to the calculated data similarly to the experimental data by calculating the total events solely from the frequency of full size fragments. We present both the total number of calculated events and corrected number of events in the result section.

\section{RESULTS}

All experiments in this paper were performed with a normal human fibroblast cell line (GM38) after growth to confluency, at which stage the cells were predominantly (more than 90\%) in the G1 or G0 stage of the cell cycle. 
Frequency of unrejoined breaks modeled as complex DSBs.

The percentage of initially induced DSBs that remained unrejoined after $16 \mathrm{hr}$ of repair at $37^{\circ} \mathrm{C}$ was measured by the use of a standard FAR assay as a function of LET for He-ions and Fe-ions (Figure 1). Two separate curves were obtained for He-ions and Fe-ions as expected due to the low LET component from high-energy delta rays in the case of the Fe ions $(17,18)$. The values of unrejoined breaks for these high LET particles were $10-20 \%$. In contrast, the unrejoined breaks after X-irradiation were only about 1-2 $\%$ of the initially induced breaks (data not shown).

Monte-Carlo simulations have suggested a wide spectrum of DSB complexity (19), with variable number of associated base damages and single-strand breaks within 20 bp of the DSB. As shown by Figure 1, the calculated frequency of complex DSBs with 8 or more associated base damages correlated well with the experimental values for frequency of unrejoined breaks for both He and Fe ions within the LET interval studied. This suggests that DSBs of high complexity are the breaks that do not rejoin. However, other criteria for DSB complexity, for example the presence of particular base damages or additional single-strand breaks, might also fit the data and is not ruled out as the cause for lack of rejoining.

Since the hybridization assay used below measures the sum of unrejoined and misrejoined DSBs, unrejoined breaks were subtracted experimentally by use of data as shown in Figure 1, and theoretically in the Monte-Carlo simulations by excluding complex DSBs with more than 8 associated base damages in the misrejoining calculations.

\section{Misrejoining depends on dose and particle type.}

For measurements of misrejoining (joining of wrong DNA ends) the hybridization assay first described by Löbrich et al. (20) was used together with the data analysis method described by Fouladi et al. (2). Cells were irradiated with single doses of 5-40 Gy for measurement of DSB induction, and doses of 10-80 Gy followed by $16 \mathrm{hr}$ repair incubation at $37{ }^{\circ} \mathrm{C}$ for measurement of misrejoining. After embedding of trypsinized cells in agarose plugs, lysis at $50{ }^{\circ} \mathrm{C}$ and restriction with NotI enzyme, the DNA was separated by pulsed-field gel electrophoresis. The DNA was then transferred onto nylon 
membranes and hybridized with a probe that detects a unique $3.2 \mathrm{Mbp}$ NotI fragment located on chromosome 21. As an example, Figure 2A shows a blot from a He-ion experiment with the full length NotI fragments on top with smears below the bands from initially induced DSBs (left lanes) or from the sum of misrejoined and unrejoined DSBs (right lanes). Figure 2B illustrates the method of analysis of each lane with the division of the signal into peak (intact NotI fragment) and smear. Observe the way this method approximates the presence of a smear of fragments of the same size as the NotI peak by a straight line connecting the smear on each side of the peak. The analysis in this paper excludes the signal above the band (NotI fragments larger than the original NotI fragment) since this signal was not sufficiently reproducible, thereby slightly underestimating the total misrejoining. The number of misrejoined plus unrejoined breaks $(\mathrm{N})$ are then calculated from the fraction of intact NotI fragments $(\mathrm{P})$ by the assumption of a Poisson distribution:

$\mathrm{N}=-\ln \mathrm{P}$

The number $\mathrm{N}$ obtained for unirradiated control cells is then subtracted from the irradiated cells within each experiment to obtain the number due to irradiation. Finally, unrejoined breaks, as estimated from Figure 1 (and similar data for X-rays and the Nions, not shown), are subtracted to arrive at the misrejoining frequency.

Independent experiments as described above were carried out in triplicate for $\mathrm{X}$ rays, $7 \mathrm{MeV} \mathrm{He}$-ions and $97 \mathrm{MeV} \mathrm{N}$-ions. In addition, a single experiment was carried out with $1 \mathrm{GeV}$ Fe-ions. The X-ray data are plotted in Figure 3 and all data together, including the high LET particle data, are plotted in Figure 4. Note the curvilinear induction of DSB misrejoining for X-rays and the somewhat more linear induction for the high LET particles.

Theoretical Monte Carlo simulations give a good fit to all the data within experimental errors when assuming $0.25 \mu \mathrm{m}$ interaction distance for misrejoining events.

To simulate the experimental results, realistic particle track calculations were performed with a full 46 chromosome model of the cell nucleus to obtain the spatial location of all DSBs as previously described (11). The rejoining probability for two DNA ends was then assumed to depend on the distance between the ends as described by a 
bell-shaped Gaussian function with a standard deviation of $\sigma \mu \mathrm{m}$. For X-rays at the doses used, the DNA ends are nearly randomly distributed within the nucleus with an average distance that diminishes with increasing dose. Thus, in this case the misrejoining probability is very low at low doses but increases with higher doses. For high LET particles, the intra-track spatial distribution does not change with dose, giving rise to a misrejoining probability component that is independent of dose.

The simulations done this way for various irradiation qualities and doses yielded a total misrejoining frequency. However, not all misrejoining events can be detected experimentally. For example, inversions within the NotI fragment itself or very small deletions generated particularly by high LET radiations are not detected. The theoretical misrejoining frequencies were therefore corrected to correspond to the experimental frequencies. For details of this correction, see Materials and Methods. It was found that by adjusting the misrejoining interacting distance $\sigma$ to $0.25 \mu \mathrm{m}$, a good fit to the X-ray data was obtained as shown in Figure 3. This interacting distance also generated simulated misrejoining yields for the high LET radiations that were in agreement with the experimental results within experimental errors (Figure 4). For comparison, the total misrejoining frequencies as obtained by the Monte-Carlo calculations are also plotted in Figure 5 for $\mathrm{He}$ ions and X-rays. As expected, the correction due to experimental limitations affects mainly the high LET results.

Due to the shapes of the misrejoining yield as a function of dose, the relative difference in misrejoining between high LET particles and X-rays increases with decreasing dose. Although experimental data were only obtained for doses above $10 \mathrm{~Gy}$, the simulated data could be extended to lower doses. Figure 6 shows Monte-Carlo simulations in the dose range of $0.1-2$ Gy using an interaction distance of $0.25 \mu \mathrm{m}$. The induction curves have a prominent linear component in this dose range for all radiations, including X-rays. The data was fitted with linear-quadratic polynomials and the linear components were used to calculate the relative biological effectiveness (RBE) for misrejoining for low doses. The RBE was found to be 31 for the He-ions, 28 for the Nions and 19 for Fe-ions. 


\section{DISCUSSION}

Nonrejoined DSBS.

We show experimental results and theoretical modeling for both nonrejoined and misrejoined DSBs after low LET and high LET ionizing radiation. Our data for nonrejoined DSBs as a function of LET for $7 \mathrm{MeV} \mathrm{He}$ ions and $1 \mathrm{GeV} / \mathrm{n}$ Fe ions (Fig 1) show that the situation for the two particles is quite different. This is understandable from the point of view that about half of the Fe ion energy is absorbed as high-energy delta rays of low LET $(17,18)$, and consequently the effective LET along the track for the Fe ions $\left(\mathrm{LET}_{10 \mathrm{~nm}}\right)$ is about half of the total LET $\left(\mathrm{LET}_{\infty}\right)$, which is what is plotted in Figure 1. The He ion energy, on the other hand, is much lower than the Fe ion energy, and high energy delta rays cannot be produced. Thus for the He ions the effective LET along the track is similar to the total LET.

Ionizing radiation can produce complex DSBs (19), with many associated base damages and single strand breaks within a multiply damaged site (MDS). The complexity of breaks increases with LET. We show in Figure 1 that there is a good correlation between non-rejoined breaks (after $16 \mathrm{hr}$ repair in the G0/G1 stage of the cell cycle) and break complexity as simulated by a Monte-Carlo approach. With the assumption that the most complex DSBs are those that do not rejoin, we find a good correlation between the experimental fraction of non-rejoined breaks and the simulated fraction of DSBs that have 8 or more associated base damages. It should be mentioned that other parameters of complexity may also successfully simulate the situation, since all types of complexity at the break site will correlate with each other. One can hypothesize that DNA ends with multiple chemical changes cannot be processed by DNA repair enzymes. However, the experiments were carried out with cells in the G0/G1 phase of the cell cycle, and do not exclude the possibility that these DSBs will be processed subsequently by mechanisms operating in the $\mathrm{S}$ or $\mathrm{G} 2$ phases of the cell cycle, for example by homologous recombination.

We have assumed that residual unrepaired DSBs at $16 \mathrm{hr}$ are solely due to DSB complexity and is independent on dose. At high doses, the possibility of saturation of repair proteins could also be considered as a cause for unrepaired breaks. However, for 
the human fibroblasts used in this study, we have previously found that DSB repair, as measured by the FAR assay, is independent of dose up to $340 \mathrm{~Gy}$ (1). Since the present study was performed in the dose range of $10-100$ Gy we do nor think saturation of repair has affected the results.

\section{Misrejoined DSBs.}

The hybridization assay used in this paper measures the breakage and rejoining of DNA ends generated within a $3.2 \mathrm{Mbp}$ restriction fragment in chromosome 21 . Restoration of the original size restriction fragment is taken as a measure of joining of correct ends. The DSBs not correctly rejoined at any time point during post-irradiation incubation consist of two components: the nonrejoined breaks and the misrejoined breaks, both of which are seen as a smear in the gels. For the human fibroblast cells used in this work, we have chosen to look at the situation $16 \mathrm{~h}$ after irradiation, since at that time we have previously established that misrejoining is essentially complete (6). To obtain the misrejoining frequency, we measure the nonrejoined breaks separately by a standard FAR assay and subtract that value from the results obtained by the hybridization assay. Nonrejoined breaks at $16 \mathrm{~h}$ after irradiation are below $2 \%$ of the DSBs originally formed for X-rays and about $10 \%$ of these originally formed for the high LET particles used in the misrejoining study (see Fig 1).

The resolution of the PFGE system will allow detection of a shift of NotI fragments corresponding to changes in size by a few hundred $\mathrm{kbp}$. Changes smaller than this limit will contribute to a smear that stretches under the intact NotI band. Such changes will still be detected by the analysis methods employed, provided that such changes are not over-represented. This is accomplished by extrapolation of the smear present on each side of the intact NotI peak by a linear function (Fig 2B). Our simulations have indicated this to be a good approximation for randomly occurring DSBs from low LET radiation. For high LET radiation, however, small changes are much more common due to generation of closely correlated DSBs. The Monte-Carlo calculations presented in this manuscript show that misrejoining by high LET radiation will be under-represented due to the inability to detect small changes by the PFGE system (Figure 5). We have taken this into account when simulating the experimental results as shown in Figure 4. 


\section{Interaction distance for misrejoining.}

The theoretical method to simulate misrejoining is based on a cell nucleus model with interphase chromosomes occupying individual domains in random coil confirmations (11). A small overlap (7\%) is present in the chromosomal domains. The spatial positions of DSBs in this model are calculated by the use of realistic track structure models and estimating both direct and indirect action on DNA. After the initial coordinates for the DSBs are known for a particular simulation in terms of charged particle type and dose, the subsequent rejoining events are simulated with the assumption that the likelihood for misrejoining depends on distance between DNA ends but is independent on break complexity. The correct ends are always the most likely to rejoin under these assumptions and will effectively be the only possibility at low doses of low LET radiation with no other nearby ends. However, for higher doses of low LET radiation and for high LET radiation at all doses, other DNA ends may be present in sufficiently proximity in the affected cells to compete for rejoining. The main adjustable parameter used to fit the calculations to the experimental data is the average interaction distance $\sigma$ in an assumed Gaussian function that describes the probability for joining as a function of distance between two arbitrary DNA ends in competition for rejoining by a non-homologous end-joining event. It was found that a good fit to the low LET and an acceptable fit to the high LET experimental results were obtained with an interaction distance $\sigma$ equal $0.25 \mu \mathrm{m}$. This interaction distance is substantially smaller than the interaction distance of $0.5 \mu \mathrm{m}$ previously found to best fit chromosomal aberration data in human lymphocytes using the same methodology (11). This discrepancy is discussed in the following two paragraphs.

For the inter-chromosomal aberration simulations performed previously (11), chromosome overlap and interaction distance were found to be interrelated. With larger overlap between chromosomes, smaller interaction distance would fit the data. It is possible that the nuclear model used is insufficiently compact with spaces and with insufficient overlap between chromosomes. A more compact model of the nucleus will provide less empty space with more chromosomal overlap, and could therefore be more realistic. A more compact model with $0.25 \mu \mathrm{m}$ interaction distance could still be 
consistent with experimental inter-chromosomal aberration frequencies but bring down the predicted intra-chromosomal aberration frequencies, which appeared to be too high in the previous study (11). A theoretical interaction distance smaller than previously suggested therefore is expected for an improved model of the nucleus.

Another possibility is that the chromosomal architecture is different in fibroblasts compared to lymphocytes. Interaction distance for any particular cell type could depend on cytoskeletal structure, nuclear size and shape. In fact, chromosome aberration yields have been reported to be higher in lymphocytes than in fibroblasts (21), consistent with a larger interaction distance in the lymphocytes. It is also not ruled out that a fraction of chromosomal aberrations are formed by other mechanisms not requiring misrejoining of two initially formed DSBs.

\section{Misrejoining as a function of LET}

The Monte-Carlo calculations in this paper build on first principles and a single adjustable parameter, the interaction distance between DSBs. It is found that the high LET data can be simulated within experimental errors using the same interaction distance as found optimal for the low LET data with no further assumptions. This suggests that the higher frequency of misrejoining seen for high LET radiation is caused by the spatial distribution of DSBs over sub-micrometer distances. However, there are likely additional factors that influence to a small extent the misrejoining frequencies. For example, misrejoining could depend on the DSB complexity. A "dirty" DSB might be repaired slower and may therefore be more available for misrejoining. Since high LET radiation produces more complex DSBs, this would predict increased misrejoining frequency for the high LET radiations. The data in figure 4 indeed do suggest that the high LET data are slightly under-predicted, and we therefore believe such theoretical assumptions could have led to an improved fit. However, there is presently no clear theoretical understanding how the relationship between DSB complexity and misrejoining should be modeled, and we do not feel such an attempt is warranted at the present level of experimental accuracy. The data as presented suggests that the closer proximity of DSBs for high LET radiations explains the higher misrejoining frequency, without ruling out the possibility that complexity of breaks plays an additional role. 
At the highest dose of $80 \mathrm{~Gy}$, we observe here approximately a two-fold higher misrejoining yield for the high-LET particles compared to X-rays. This is in apparent conflict with our earlier study carried out at $80 \mathrm{~Gy}$ (6), where we reported a yield that was nearly independent of LET. We think the main difference is in the data analysis of the hybridization profiles, where we previously considered all DNA in the peak area of the blots to belong to full size intact restriction fragments, while in the present study we have improved the estimation of the smear area by extrapolation under the peak as illustrated in Figure 2B. The previously used method underestimates the misrejoining frequency, particularly for high LET radiation, since such radiation produces a large yield of smaller changes that will not be distinguished from intact full size fragments. We therefore believe the present data is more accurate. We note however, as illustrated in Figure 5, that the improved experimental protocol still underestimates the high LET misrejoining frequency due to inability to detect all events that involve small DNA segments.

The results suggest that the relative biological efficiency (RBE) for high LET radiation with regard to misrejoining frequency increases with decreasing dose, and the Monte-Carlo calculations tentatively suggest that the RBE could be in the range of 18 31 at biologically significant doses of $0.1-2$ Gy. In addition, both theory and experimental results show that the high-energy highly charged $\mathrm{Fe}$ ions are intermediate in effects compared to He ions and X-rays. This result is relevant to space travel, since such HZE ions are present as an important component of the galactic cosmic rays.

\section{ACKNOWLEDGEMENTS}

This work was supported by grants from the National Institutes of Health (CA80207) to P.K. Cooper, order No. W-18,265 from the U.S. National Aeronautics and Space Administration (NASA) to the NSCORT in Radiation Health, and by the Office of Biological and Environmental Research, Office of Energy Research, U. S. Department of Energy Office of Science, under Contract No. DE-AC03-76SF00098. 


\section{REFERENCES}

1. M. Löbrich, B. Rydberg, and P. K. Cooper. Repair of X-ray-induced DNA doublestrand breaks in specific Not I restriction fragments in human fibroblasts: joining of correct and incorrect ends. Proc Natl Acad Sci U S A 92: 12050-4 (1995).

2. B. Fouladi, C. A. Waldren, B. Rydberg, and P. K. Cooper. Comparison of repair of DNA double-strand breaks in identical sequences in primary human fibroblast and immortal hamster-human hybrid cells harboring a single copy of human chromosome 11. Radiat Res 153: 795-804. (2000).

3. T. Radivoyevitch, D. G. Hoel, A. M. Chen, and R. K. Sachs. Misrejoining of double-strand breaks after $\mathrm{X}$ irradiation: Relating moderate to very high doses by a Markov model. Radiat Res 149: 59-67 (1998).

4. D. R. Cox, M. Burmeister, E. R. Price, S. Kim, and R. M. Myers. Radiation hybrid mapping: a somatic cell genetic method for constructing high-resolution maps of mammalian chromosomes. Science 250: 245-50 (1990).

5. A. M. Kellerer and H. H. Rossi. A generalized formulation of dual radiation action. Radiat. Res. 75: 471-488 (1978).

6. M. Löbrich, P. K. Cooper, and B. Rydberg. Joining of Correct and Incorrect DNA Ends at Double-Strand Breaks Produced by High-Linear Energy Transfer Radiation in Human Fibroblasts. Radiation Research 150: 619-626 (1998).

7. B. Rydberg. Radiation-induced heat-labile sites that convert into DNA doublestrand breaks. Radiat Res 153: 805-812 (2000).

8. B. Stenerlow, K. H. Karlsson, B. Cooper, and B. Rydberg. Measurement of Prompt DNA Double-Strand Breaks in Mammalian Cells without Including HeatLabile Sites: Results for Cells Deficient in Nonhomologous End Joining. Radiat Res 159: 502-10 (2003).

9. M. Lobrich, M. Kuhne, J. Wetzel, and K. Rothkamm. Joining of correct and incorrect DNA double-strand break ends in normal human and ataxia telangiectasia fibroblasts. Genes Chromosomes Cancer 27: 59-68 (2000).

10. M. Kühne, K. Rothkamm, and M. Löbrich. No dose-dependence of DNA doublestrand break misrejoining following alpha-particle irradiation. International Journal of Radiation Biology 76: 891-900 (2000).

11. W. R. Holley, I. S. Mian, S. J. Park, B. Rydberg, and A. Chatterjee. A Model for Interphase Chromosomes and Evaluation of Radiation-Induced Aberrations. Radiat Res 158: 568-580. (2002).

12. C. Zeitlin, L. Heilbronn, and J. Miller. Detailed characterization of the 1087 $\mathrm{MeV} /$ nucleon iron-56 beam used for radiobiology at the alternating gradient synchrotron. Radiat Res 149: 560-9 (1998). 
13. B. Rydberg, L. Heilbronn, W. R. Holley, M. Lobrich, C. Zeitlin, A. Chatterjee, and P. K. Cooper. Spatial Distribution and Yield of DNA Double-Strand Breaks Induced by 3- $7 \mathrm{MeV}$ Helium Ions in Human Fibroblasts. Radiat Res 158: 32-42. 7587 \& volume $=158$ \&issue $=1$ \&page $=32$ (2002).

14. D. Blöcher, M. Einspenner, and J. Zajackowski. CHEF electrophoresis, a sensitive technique for the determination of DNA double-strand breaks. Int $J$ Radiat Biol 56: 437-448 (1989).

15. D. Blöcher. In CHEF electrophoresis a linear induction of dsb correspond to a nonlinear fraction of extracted DNA with dose. Int J Radiat Biol 57: 7-12 (1990).

16. M. Löbrich, B. Rydberg, and P. K. Cooper. DNA double-strand breaks induced by high-energy neon and iron ions in human fibroblasts. II. Probing individual notI fragments by hybridization. Radiat Res 139: 142-51 (1994).

17. W. E. Wilson and T. L. Criswell. Microscopic track structure of equal-LET heavy ions. Adv Space Res 6: 75-81 (1986).

18. A. Chatterjee and T. H. Borak. Physical and biological studies with protons and HZE particles in a NASA supported research center in radiation health. Phys Med 17 Suppl 1: 59-66 (2001).

19. W. R. Holley and A. Chatterjee. Clusters of DNA damage induced by ionizing radiation: formation of short DNA fragments. I. Theoretical modeling. Radiat Res 145: 188-199 (1996).

20. M. Löbrich, S. Ikpeme, and J. Kiefer. Measurement of DNA double-strand breaks in mammalian cells by pulsed-field gel electrophoresis: A new approach using rarely cutting restriction enzumes. Radiat Res 138: 186-192 (1994).

21. P. Virsik-Peuckert, M. Rave-Frank, U. Langebrake, and H. Schmidberger. Differences in the yields of dicentrics and reciprocal translocations observed in the chromosomes of irradiated human skin fibroblasts and blood lymphocytes from the same healthy individuals. Radiat Res 148: 209-15 (1997). 


\section{FIGURE LEGENDS.}

Figure 1. Percentage of unrejoined breaks plotted against LET for He and Fe ions. The open triangles represent data for helium ions of different LET values (three independent experiments performed with doses $25 \mathrm{~Gy}, 80 \mathrm{~Gy}$ and $100 \mathrm{~Gy}$ ). The solid black triangles represent data for iron ions at two different LET values at $80 \mathrm{~Gy}$. The broken lines and the solid line are the results from the theoretical model for percentage of DSBs with $\mathrm{N}$ or more base damages. The data and the theoretical results are in agreement when $\mathrm{N}=8$, ie. with 8 or more base damages. For $\mathrm{N}=6$ or $\mathrm{N}=10$ there is no correlation with the data.

Figure 2. Panel A: Typical gel from which misrejoining was calculated. The bands are the intact 3.2 Mbp NotI fragments. The smear below the bands originates from broken or misrejoined fragments. Panel B: Quantitation of band and smear intensities. Shown is a scan of the 20 Gy induction lane in panel A with dotted lines defining the background intensity below the smear and the dividing line between peak and smear. Panel $\mathbf{C}$ and D: Rational for using a straight line to divide peak and smear areas. An idealized distribution in panel $\mathrm{C}$ is convoluted with a Gaussian function in panel $\mathrm{D}$ to simulate the resolution of the gel. The step function representing the smear in panel $\mathrm{C}$ is transformed into a sigmoidal curve in D. A straight line dividing the peak and smear is a good approximation in this case.

Figure 3. Experimental misrejoining yields induced by X-rays compared to calculated misrejoining yields for four different interaction distances $\sigma$. Calculations with $0.25 \mu \mathrm{m}$ interaction distance fits the data well, while $0.20 \mu \mathrm{m}, 0.30 \mu \mathrm{m}$ or $0.50 \mu \mathrm{m}$ are not consistent with the data. Error bars indicate standard error of the mean from 3 independent experiments.

Figure 4. DNA misrejoining yield as a function of dose for X-rays, Fe ions (150 $\mathrm{keV} / \mu \mathrm{m}), \mathrm{N}$-ions $(97 \mathrm{keV} / \mu \mathrm{m})$ and $\mathrm{He}$ ions $(70 \mathrm{keV} / \mu \mathrm{m})$. Symbols represent experimental data, and lines theoretical Monte-Carlo calculations with the DSB 
interaction distance set to $0.25 \mu \mathrm{m}$. Error bars indicate standard error of the mean from three independent experiments. The Fe ion data are from a single experiment.

Figure 5. Calculated misrejoining yields for $0.25 \mu \mathrm{m}$ interaction distance before and after correction to experimentally detectable yields. Note that many calculated misrejoining events are not detected with the experimental approach after high LET irradiation.

Figure 6. Calculated misrejoining yields in the dose range of $0.1-2$ Gy. Insert: Same data with expanded vertical scale to see the X-ray data more clearly. The points represent calculated Monte-Carlo calculations and the lines are fitted second degree polynomials. Observe the much higher misrejoining frequencies for the high-LET ions compared to Xrays in this dose range. The linear components of the fitted polynomials indicate an RBE of 31 for the He ions, 28 for the $\mathrm{N}$ ions and 19 for the $\mathrm{Fe}$ ions in the low dose region. 
Rydberg et al., Figure 1

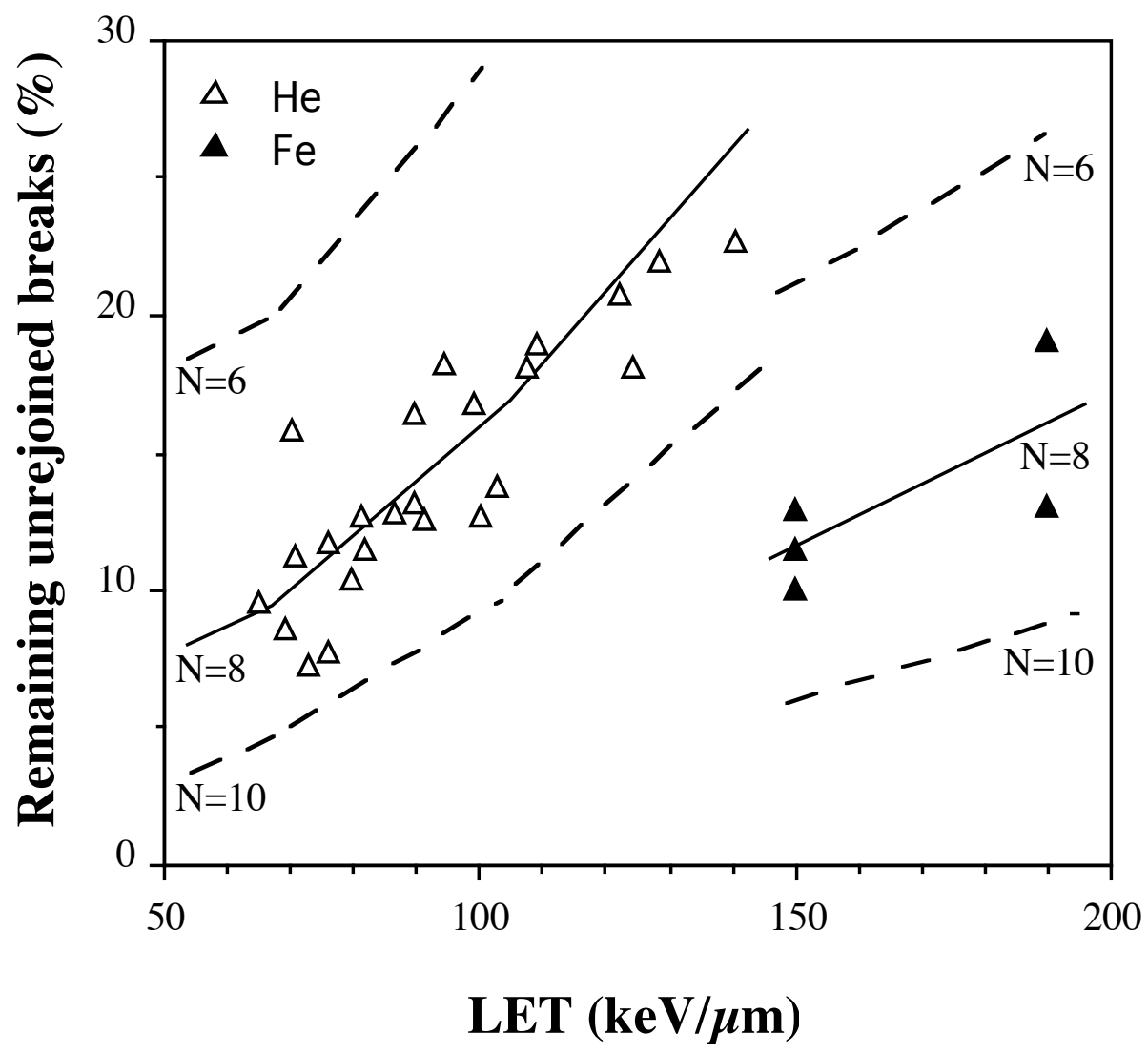


Rydberg et al., Figure 2A

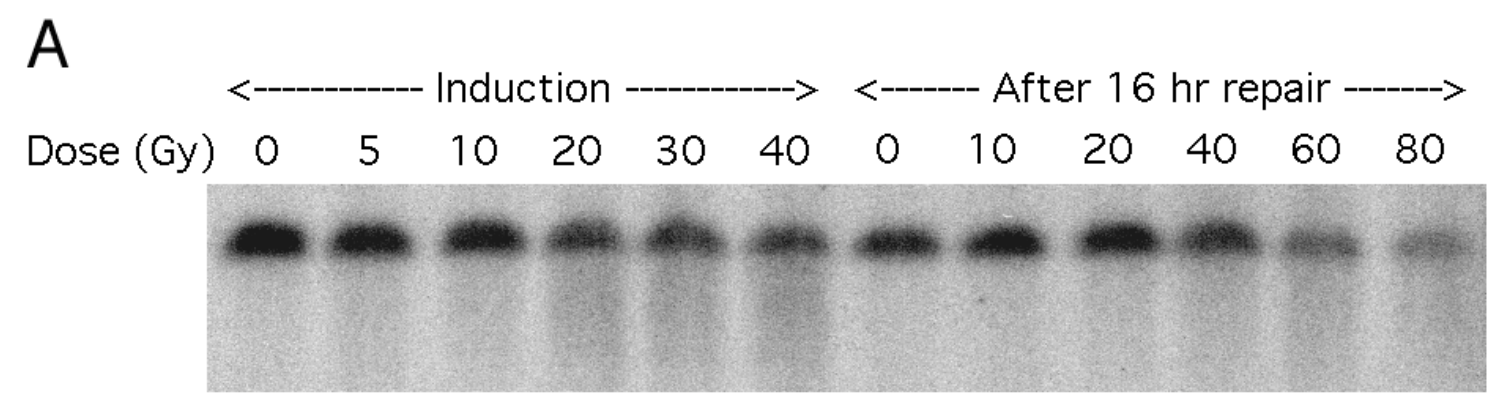


Rydberg et al., Figure 2B

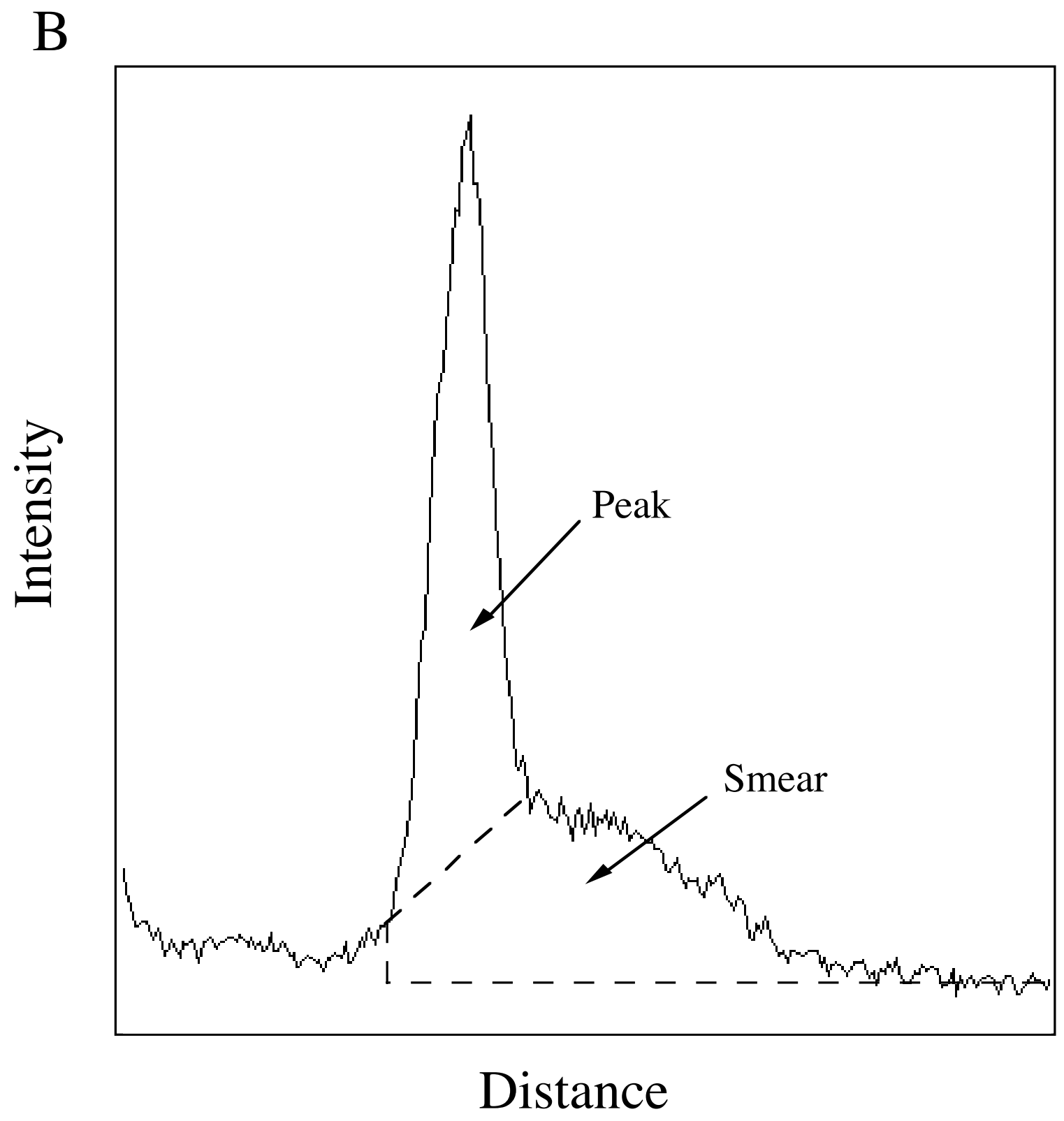


Rydberg et al. Figure 2C and 2D

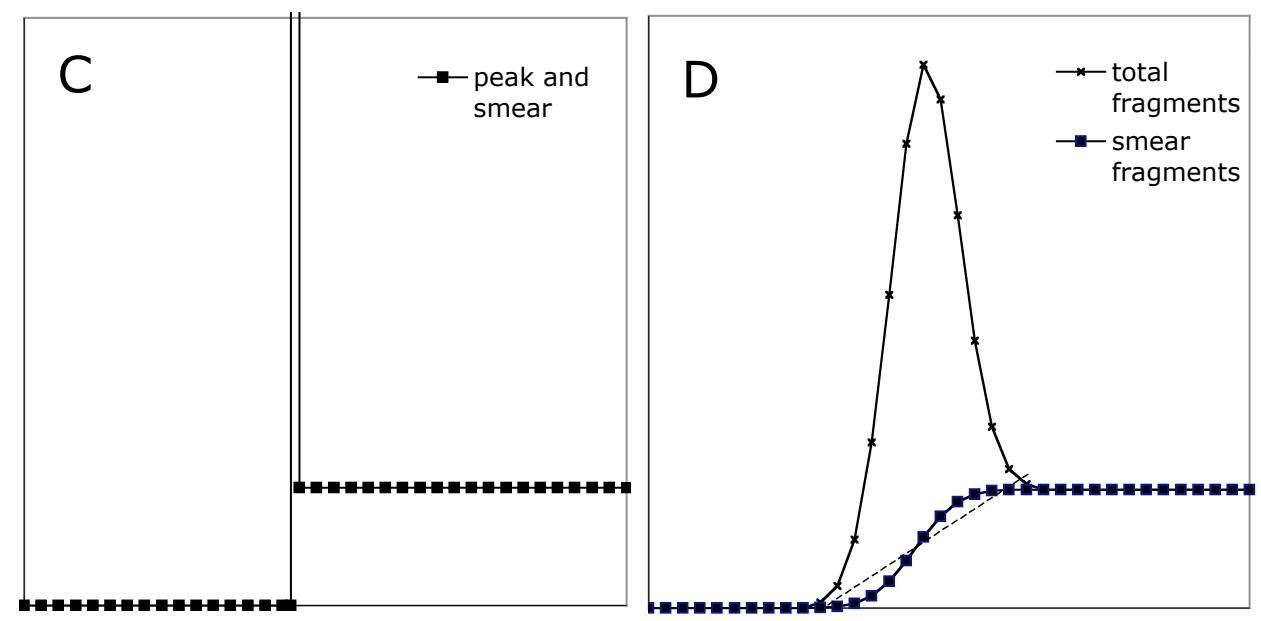


Rydberg et al., Figure 3

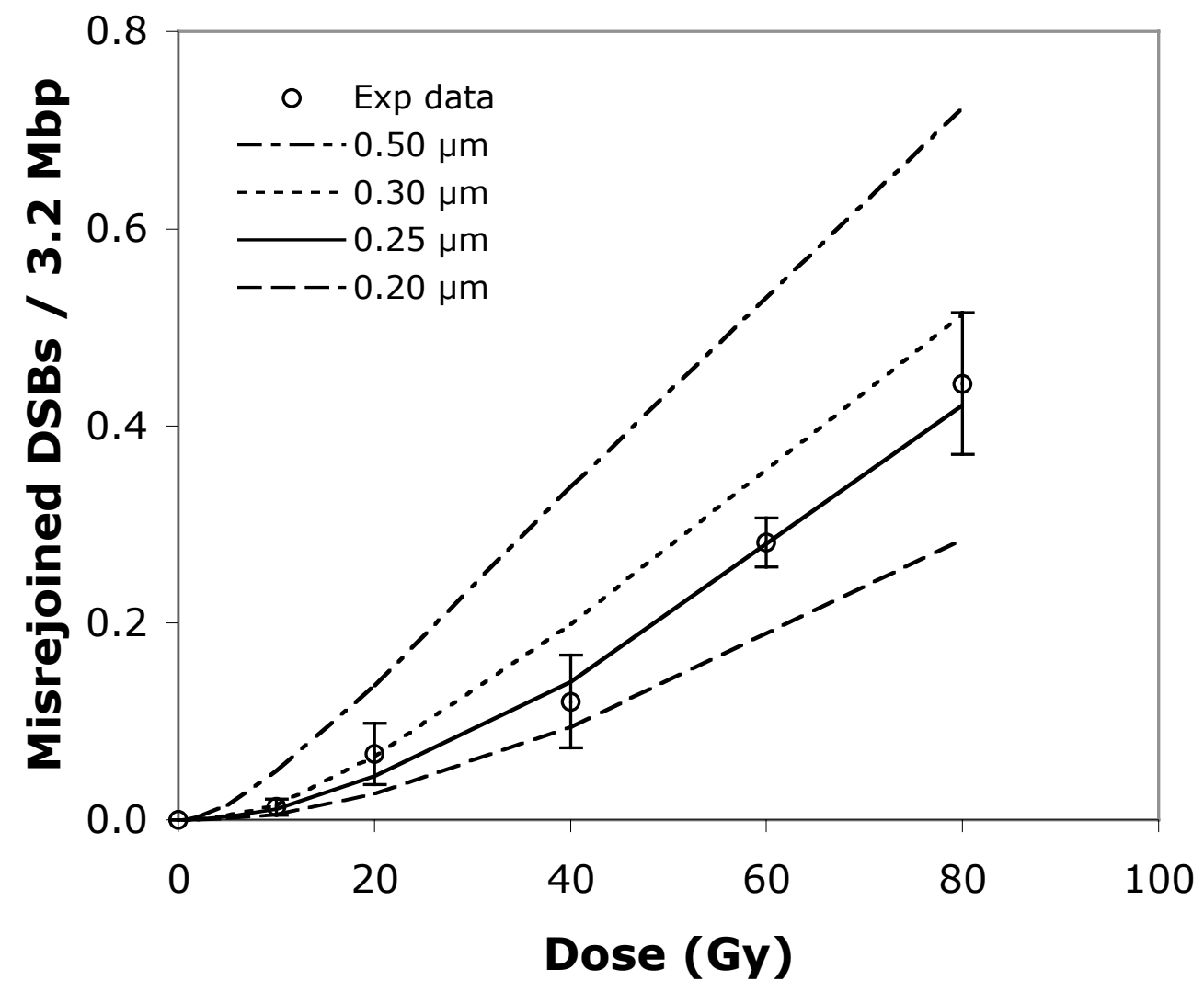


Rydberg et al., Figure 4

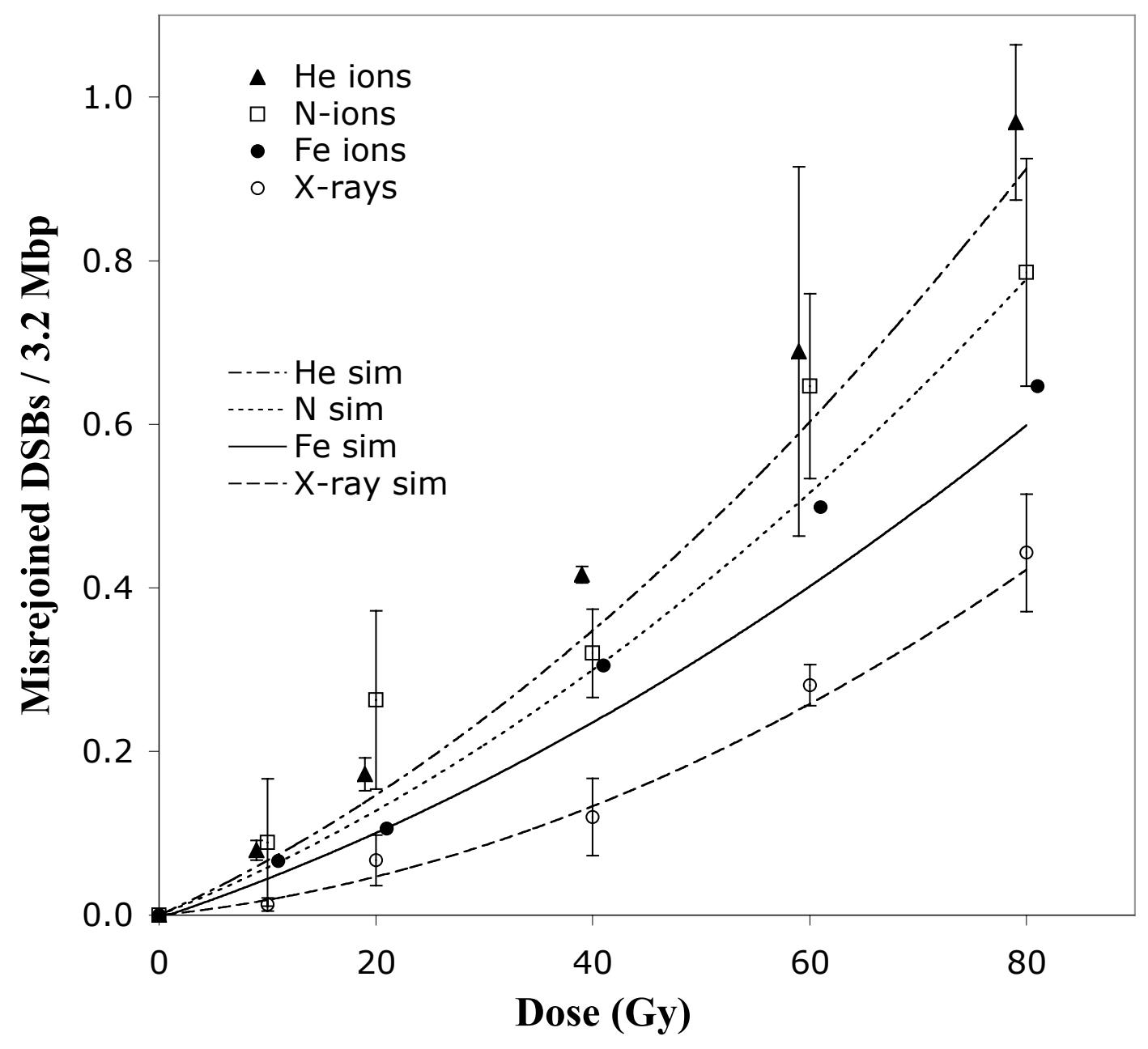


Rydberg et al., Figure 5

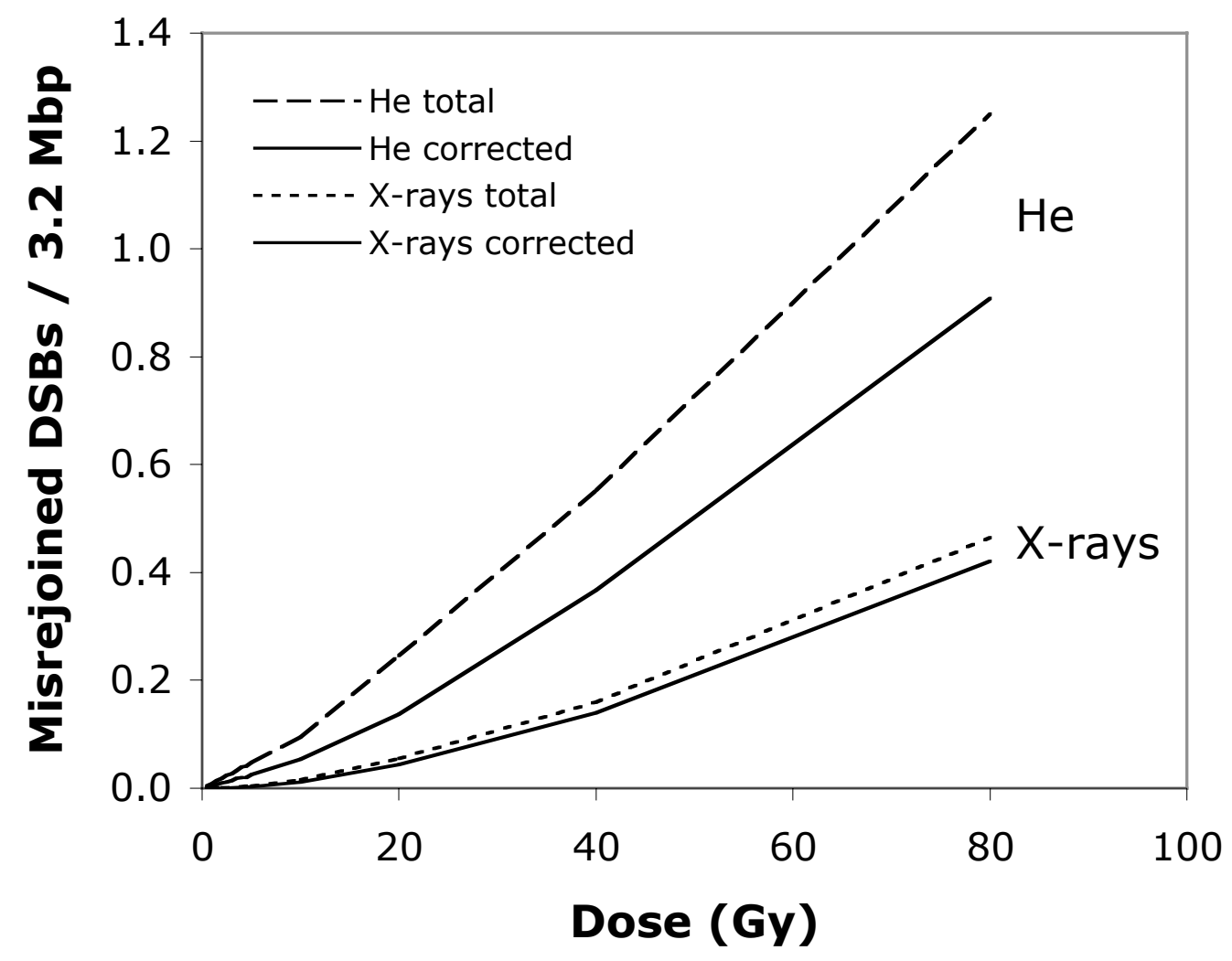


Rydberg et al. Figure 6

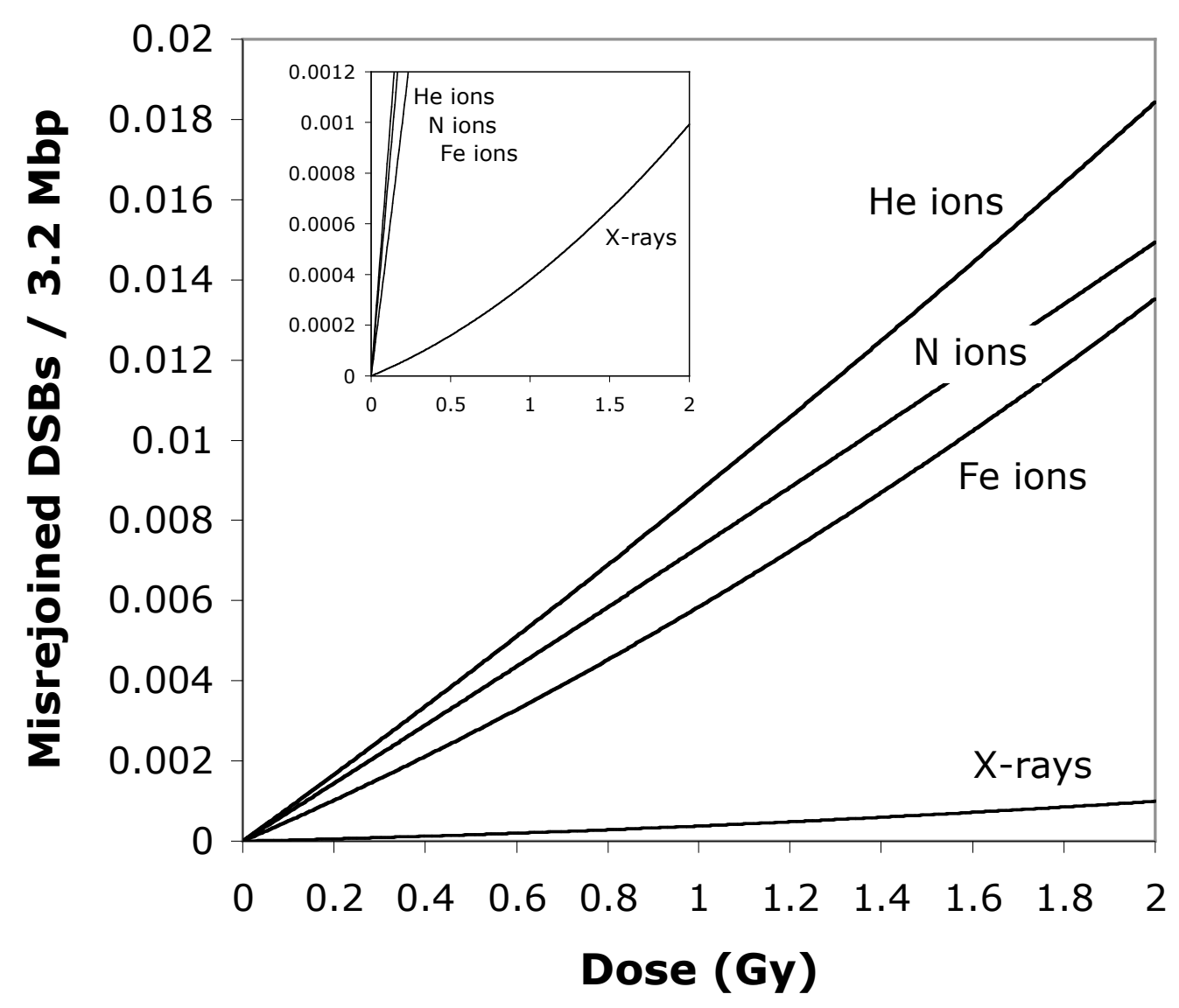

